\title{
Broadband Dual-Polarized Single-Layer Reflectarray Antenna with Independently Controllable 1-Bit Dual Beams
}

This paper was downloaded from TechRxiv (https://www.techrxiv.org).

\section{LICENSE}

CC BY 4.0

\section{SUBMISSION DATE / POSTED DATE}

$21-07-2020$ / 26-07-2020

\section{CITATION}

Yin, Jiexi; Lou, Qun; Wang, Haiming; Chen, Zhining; Hong, Wei (2020): Broadband Dual-Polarized SingleLayer Reflectarray Antenna with Independently Controllable 1-Bit Dual Beams. TechRxiv. Preprint. https://doi.org/10.36227/techrxiv.12681989.v1

$\mathrm{DOI}$ 


\title{
Broadband Dual-Polarized Single-Layer Reflectarray Antenna with Independently Controllable 1-Bit Dual Beams
}

\author{
Jiexi Yin, Member, IEEE, Qun Lou, Member, IEEE, Haiming Wang, Member, IEEE, \\ Zhi Ning Chen, Fellow, IEEE, and Wei Hong, Fellow, IEEE.
}

\begin{abstract}
A broadband dual-polarized single-layer 1-bit unit cell is proposed for achieving the independently controllable dualbeam reflectarray antenna. The unit cell independently provides two-state phase compensation for two orthogonally linearlypolarized waves. The 180-degree reflective phase difference between the two states is achieved by tuning the magnetic resonance of State 0 and the electrical resonance of State 1 . With its two resonances close to each other, the unit cell has a reflective phase difference of $180 \pm 20$ degrees between two states over a broad bandwidth of 27.2-51.1 GHz. The cross-polarization levels of below $-30 \mathrm{~dB}$ ensure the high isolation between two polarizations. Using the proposed dual-polarized unit cells, a 1-bit dual-beam reflectarray antenna is designed and excited by a dualpolarized horn to show the ability of independently controlling two orthogonally linearly-polarized waves. At $33 \mathrm{GHz}$, the beams direct to -15 degrees and 20 degrees for the feeding of horizontally and vertically polarized port, respectively. The $1.5-\mathrm{dB}$ gain bandwidth is greater than $20 \%$ for both polarizations. The proposed dual-polarized reflectarray antenna with independently controllable 1-bit dual beams provides an alternative design for the multiuser multiple-input multiple-output applications.
\end{abstract}

Index Terms-Dual-polarized antennas, millimeter wave antennas, multibeam antennas, reflectarrays.

\section{INTRODUCTION}

W ITH the demand for high spectral efficiency, the multiuser multiple-input and multiple-output (MU-MIMO) system is widely studied and gradually applied in the fifthgeneration (5G) mobile communication systems [1], [2]. The MU-MIMO system needs dynamic multiple beams with low interference and high gain to guarantee reliable millimeterwave (mmWave) communications. Therefore, as an important

Manuscript received June 15, 2020; revised

Date of publication ; date of current supported in part by the National Key R\&D Program of China under Grant 2020YFB1804901, the National Natural Science Foundation of China under Grants 61671145 and 61960206006, and the Key R\&D Program of Jiangsu Province of China under Grant BE2018121.

J. X. Yin is with the State Key Laboratory of Millimeter Waves and the School of Information Science and Engineering, Southeast University, Nanjing 210096, China (e-mail: yinjiexi@ seu.edu.cn).

Q. Lou and Z. N. Chen are with the Department of Electrical and Computer Engineering, National University of Singapore, Singapore 117583 (e-mail: qlou@ieee.org; eleczn@nus.edu.sg).

H. M. Wang and W. Hong are with the State Key Laboratory of Millimeter Waves and the School of Information Science and Engineering, Southeast University, Nanjing 210096, China, and with the Purple Mountain Laboratories, Nanjing 211111, China (e-mail: hmwang@seu.edu.cn; weihong@seu.edu.cn). (Corresponding author: Haiming Wang.)

Color versions of one or more of the figures in this paper are available online at http://ieeexplore.ieee.org.

Digital Object Identifier part of the MU-MIMO system, antennas with multiple independently controlled beams, high isolation, and high gain are preferred.

The metasurfaces have been widely studied in recent years and have presented many functions, such as invisibility [3], spatial reuse [4], and polarization reuse [5], [6]. The reflective metasurface with the ability of polarization reuse has the potential to independently control multiple beams with different polarizations and beam directions, which can achieve polarization diversity and spatial diversity for MUMIMO systems. Some independently controllable dual-beam dual polarization reflectarray antennas are presented using polarization reused reflective metasurface [7]-[13]. The key issue to realize independently controllable dual-beam dualpolarized metasurfaces is the design of dual-polarized unit cells, which can independently provide phase control for different polarized waves.

So far, several types of unit cells are presented to realize independent phase control for different polarizations. For two orthogonal linear polarizations, the multi-layer patches [7] and crossed dipoles [10], [11] structure are used to design independently controllable dual-polarized unit cells. Changing orthogonal geometrical dimensions can independently control phase compensation for horizontally polarized waves and vertically polarized waves, respectively. The single-layer square patch loaded with two delay lines can also be used to independently control reflective phases, which has reconfigurable potential by changing the length of the two delay lines [8]. Furthermore, for dual circular polarizations, the Berry phase compensation and dynamic phase compensation can be implemented to achieve the independently controllable dual-circularly-polarized unit cells with wide working bandwidth [12], [13], but the method is difficult to realize for linearly polarized unit cells.

The MU-MIMO system needs the ability of dynamic multiple-beam control. Considering the complexity of the controller, the unit cell with 1-bit phase compensation is preferred [14], [15]. The unit cell should also feature broadband, low cross-polarization level, simple structure, and easy loading for reconfigurable components. The broadband unit cell with low cross-polarization level ensures the bandwidth and the isolation of the reflectarray antenna. The simple structure, such as the single-layer structure, simplifies further circuit design when loading with reconfigurable components. As one of the classic structures, the single-layer square patch with two delay lines is used to realize dual-polarized unit cells for dynamic 
beams control, which has low cross-polarization, single-layer structure, and easy integration of reconfigurable components. For example, a square structure with two delay lines has been developed, and it achieves the reconfigurable dual-polarized 1-bit unit cell with PINs on the delay lines in [16].

In this work, a broadband dual-polarized 1-bit unit cell is proposed to achieve an independently controllable dualbeam reflectarray antenna. The unit cell is designed on the single-layer substrate, which features low cross-polarization level and broad bandwidth of 180-degree phase difference between two states. The unit cell can independently control reflective phases of the horizontally polarized waves and the vertically polarized waves, so the two independent beams for their respective polarization can be generated using a single-layer reflective metasurface. In the designed reflectarray antenna, a dual-polarized horn antenna with two ports is utilized to simulate two ports of the MU-MIMO system. As an example, the reflective beam directions are designed at 15-degree and 20-degree elevation angles when respectively excited by two ports of the dual-polarized horn. The proposed single-layer reflective metasurface also has the potential to realize independently dual-polarized dual-beam scanning using four electronic switches instead of four stubs on the 1-bit unit cells.

The rest of this paper is organized as follows. A dualpolarized 1-bit unit cell is proposed, the principles of operating and independent control are presented in Section II. Section III describes the 1-bit dual-beam dual-polarized reflectarray antenna design with proposed unit cells. Simulation and measurement results are shown and compared with those in references in Section IV. Finally, conclusions are drawn in Section V.

\section{Dual-Polarized 1-Bit Unit Cell}

In this study, the electromagnetic (EM) waves in which the E-field paralleling to the $x$-axis are assumed as horizontally polarized waves and the EM waves in which the E-field paralleling to the $y$-axis are assumed as vertically polarized waves.

\section{A. Structure of the Unit Cell}

The structure of the proposed independently controllable dual-polarized 1-bit unit cell is shown in Fig. 1, and the prototype is a dependently controllable dual-polarized unit cell in [17]. The essential components of the unit cell include a $2 \times 2$ square patch array is printed on a piece of grounded dielectric substrate. The square patch array is positioned in the center of the unit cell. There are two dark grey stubs, denoted by $S_{x}$, along with the longitudinal slot and between the square patch array, which is symmetric with the center of the unit cell. Two stubs $S_{x}$ are used to connect the square patches along the $x$ direction, which is utilized to control the phase compensation for horizontally polarized waves. For horizontally polarized waves, the unit cells with and without the two dark grey stubs present State 1 and State 0, respectively. The two light grey stubs, denoted by $S_{y}$, are along with the transverse slot between the square patch array. These light grey stubs are similar with the two dark grey stubs and are utilized to connect the square patches along the $y$-direction, which can control the phase compensation for vertically polarized waves.

The unit cell structure is rotationally symmetric. The dark grey stubs and light grey stubs are separately used to independently control horizontally and vertically polarized waves, respectively. The symmetric structure achieves the same response for the two polarizations. Thus, the horizontally polarized incident waves will be discussed as an example.

\section{B. Principle of 1-Bit Phase Compensation}

For the proposed unit cell, the principle of 1-bit phase compensation can be analyzed by the magnetic resonance and the electric resonance of the states. For State 0 , the unit cell acts as a magnetic wall, which provides a 0 -degree reflective phase. For State 1, the unit cell works as an electric wall, which provides a 180 -degree reflective phase.

Without loss of generality, the incident waves are assumed as horizontal polarization. As shown in Fig. 2, for State 0 of $S_{x}$, currents on the patch, currents on the ground, and displacement currents between the patch and ground form an electric loop, the loop parallels to the horizontal direction and brings a magnetic resonance, therefore the unit cell provides a 0 -degree phase compensation for horizontal waves. For State 1 of $S_{x}$, the whole patch works like an electric dipole, the dipole parallels to the horizontal direction and brings an electric resonance, therefore the unit cell provides a 180-degree phase compensation for horizontal waves.

Because the trend of the reflective phase for the two states against the frequency is similar, if the magnetic resonance frequency for State 0 is close to the electric resonance frequency for State 1, the unit cell can realize wide bandwidth for 1-bit phase compensation with an approximate 180-degree reflective
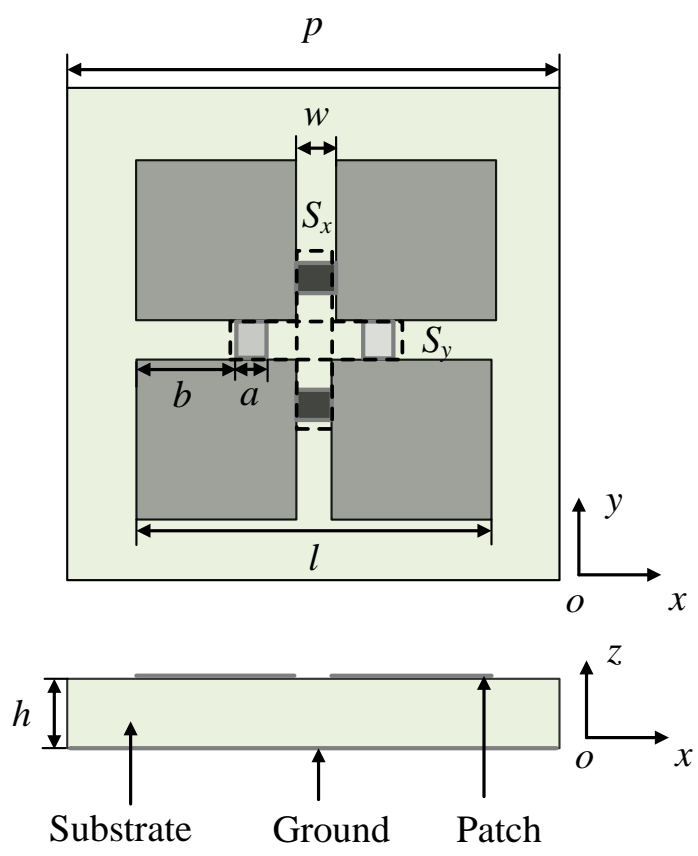

Fig. 1. Geometry of the proposed dual-polarized 1-bit unit cell. 


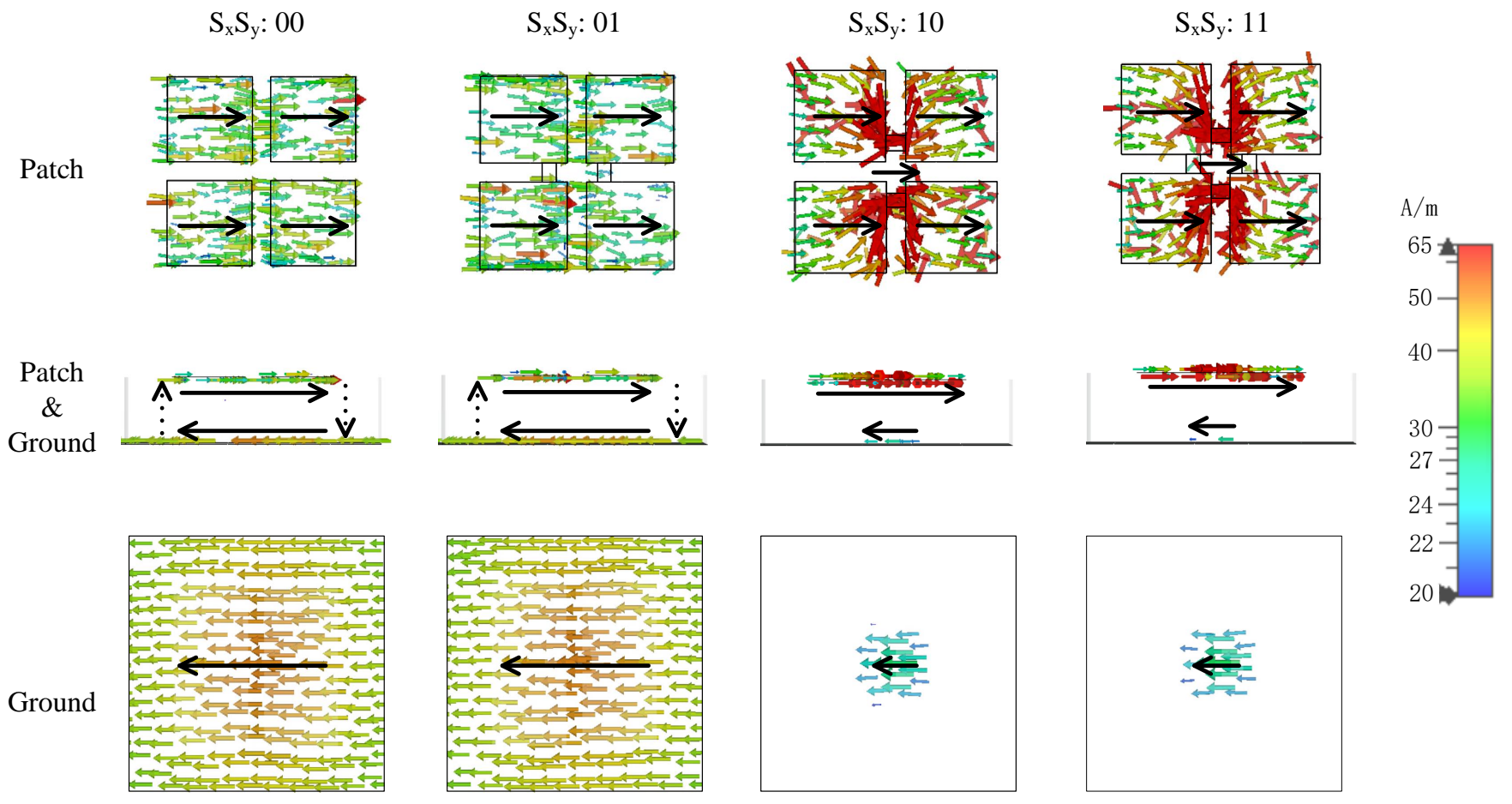

Fig. 2. Current distributions of the patch and the ground for different states, under horizontally polarized incident waves.

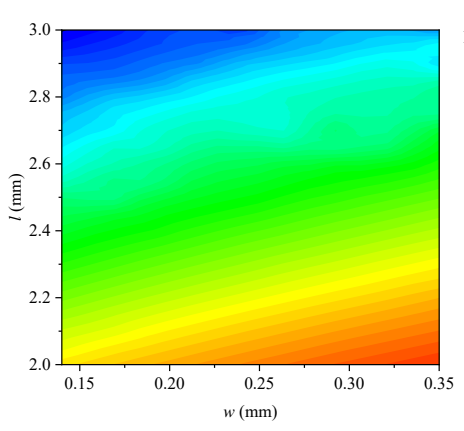

(a)

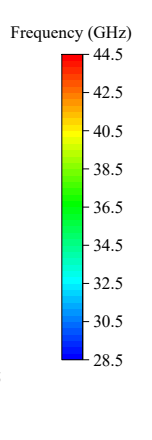

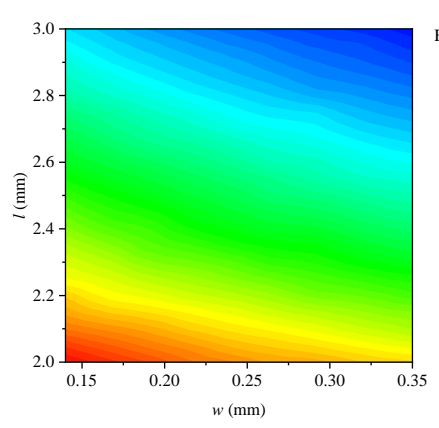

(b)

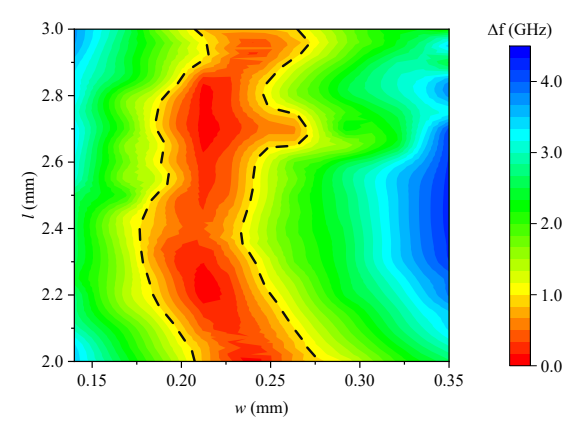

(c)

Fig. 3. The relationship between the parameters of the unit cell and the resonances, under horizontally polarized incident waves: (a) magnetic resonance frequency of State 0 for different values of $w$ and $l$, (b) electric resonance frequency of State 1 for different values of $w$ and $l$, and (c) frequency difference between the two resonances, under the same values of $w$ and $l$, where the Red Portion within two dashed lines means the frequency difference of two resonances less $1.0 \mathrm{GHz}$.

phase difference. In addition, for State 1 of $S_{y}$, less current is on the stubs for $S_{y}$; on the square patch array, the sum of the currents is almost along the $x$-direction, as shown in Fig. 2. Therefore, high polarization isolation of the unit cell can be realized. Although the discussion of the unit cell is for horizontally polarized incident waves, a similar analysis also can be used for vertically polarized incident waves.

From the analysis above, to realize the 180-degree reflective phase difference between two states, the frequency of the magnetic resonance and the electric resonance should be close enough. That means the equivalent electric loop's length for State 0 should be the twice of the equivalent electric current length for State 1 . The length of the patch $l$, the wide of the cross slot $w$, and the thickness of the substrate $h$ determine the frequency of the magnetic resonance and the electric resonance. The thickness of the substrate usually is limited by the Printed Circuit Board (PCB) process, so the length of the patch and the wide of the cross slot should be carefully designed and optimized. The relationship between the parameters and the resonance frequencies are presented in Fig. 3. The Taconic TLY with $\varepsilon_{r}=2.2$ and $h=1.016 \mathrm{~mm}$ is used as the substrate, and the design and analysis are in Q-band for the mmWave communications.

To reveal the relationship between the parameters and help the unit cell design, the two resonance frequencies along with the changing of two parameters are shown in Figs. 3(a) and 3(b). In Fig. 3(a), the magnetic resonance shifts to a higher frequency as either $w$ increases or $l$ decreases. However, in Fig. 3(b), the electric resonance shifts to a higher frequency when either $w$ or $l$ decreases. The opposite frequency trend of $w$, will lead to an intersection between frequencies for the magnetic resonance and the electric resonance. The frequency 
TABLE I

Parameters of the Proposed Unit Cell.

\begin{tabular}{cccccc}
\hline \hline Par. & Value $(\mathrm{mm})$ & Par. & Value $(\mathrm{mm})$ & Par. & Value $(\mathrm{mm})$ \\
\hline$p$ & 3.9 & $h$ & 1.016 & $a$ & 0.19 \\
\hline$b$ & 0.79 & $l$ & 2.46 & $w$ & 0.24 \\
\hline \hline
\end{tabular}

TABLE II

STATES OF $S_{x}$ AND $S_{y}$ With THEIR PHASE COMPENSATION FOR Horizontally Polarized and Vertically Polarized WaVes.

\begin{tabular}{ccc}
\hline \hline$S_{x} S_{y}$ & H-Pol & V-Pol \\
\hline 00 & 0 deg & 0 deg \\
\hline 01 & 0 deg & $180 \mathrm{deg}$ \\
\hline 10 & $180 \mathrm{deg}$ & $0 \mathrm{deg}$ \\
\hline 11 & $180 \mathrm{deg}$ & $180 \mathrm{deg}$ \\
\hline \hline
\end{tabular}

differences of the magnetic resonance and the electric resonance under different values of $w$ and $l$ are presented in Fig. 3(c), and it is obvious that there is a Red Portion in the center of the figure which indicates the frequencies of magnetic resonance and electric resonance are very close. As shown in Figs. 3(a) and 3(b), by choosing parameters $w$ and $l$ within this Red Portion, the proposed unit cell can achieve an approximate 180-degree reflective phase difference for two states at an arbitrary frequency from $30 \mathrm{GHz}$ to $42 \mathrm{GHz}$. For example, $w=2.46 \mathrm{~mm}$ and $l=0.24 \mathrm{~mm}$ are chosen for the 1-bit unit cell design which make an approximate 180-degree reflective phase difference at $36.5 \mathrm{GHz}$. Other parameters of the unit cell are shown in Table I.

\section{Independent Control of the Dual-Polarized Unit Cell}

Based on the analysis above, the two states of $S_{x}$ and $S_{y}$ can provide broadband 1-bit phase compensation for the horizontally polarized waves and the vertically polarized waves, respectively. The states' independent control of $S_{x}$ and $S_{y}$ guarantees independent 1-bit phase control for two polarized waves. Furthermore, as shown in Fig. 2, the current distributions are almost the same for the same states of $S_{x}$ and different states of $S_{y}$ under horizontally polarized incident waves. That means the states of $S_{y}$ hardly influence the reflective phases for horizontally polarized waves. For vertically polarized waves, it easily deduces that the states of $S_{x}$ hardly influence the reflective phases, and the phases can be independently controlled by $S_{y}$. Therefore, by controlling of $S_{x}$ and $S_{y}$, the independent 1-bit phase control for two orthogonally linearly-polarized incident waves can be realized for the proposed unit cell.

The desired reflective phases for different states of $S_{x}$ and $S_{y}$ are listed in Table II. These states present four kinds of states for the proposed unit cell, as shown in Fig. 2, and the independent phase control for two polarizations can be achieved using these states. The simulated performances of the unit cells are presented in Fig. 4 and Fig. 5. Under horizontally polarized incident waves, the states of $S_{y}$ has little effect for the reflective phase, as shown in Fig. 4, which corresponds to the current analysis of Fig. 2. The 1-bit reflective phase for horizontally polarized waves can independently control from

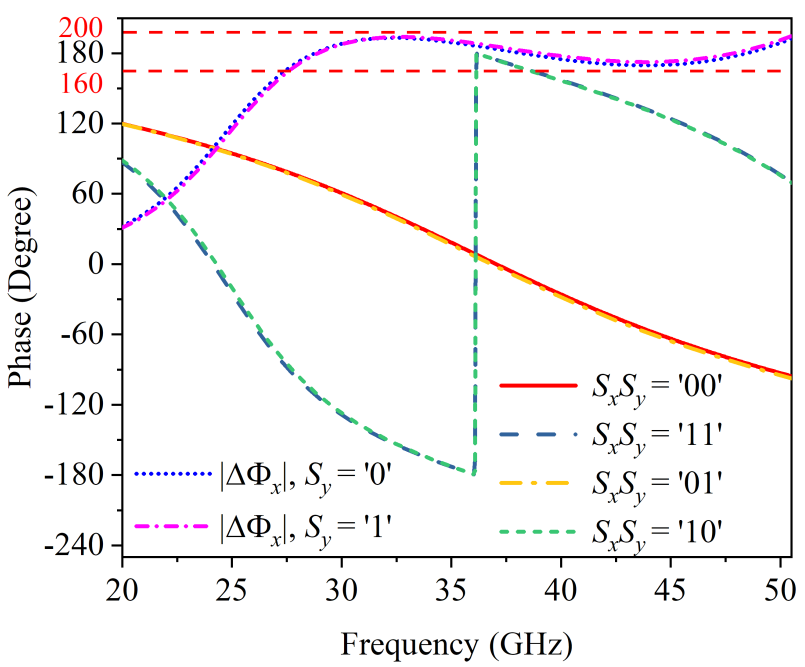

Fig. 4. Reflective phases for different states, under horizontally polarized waves.

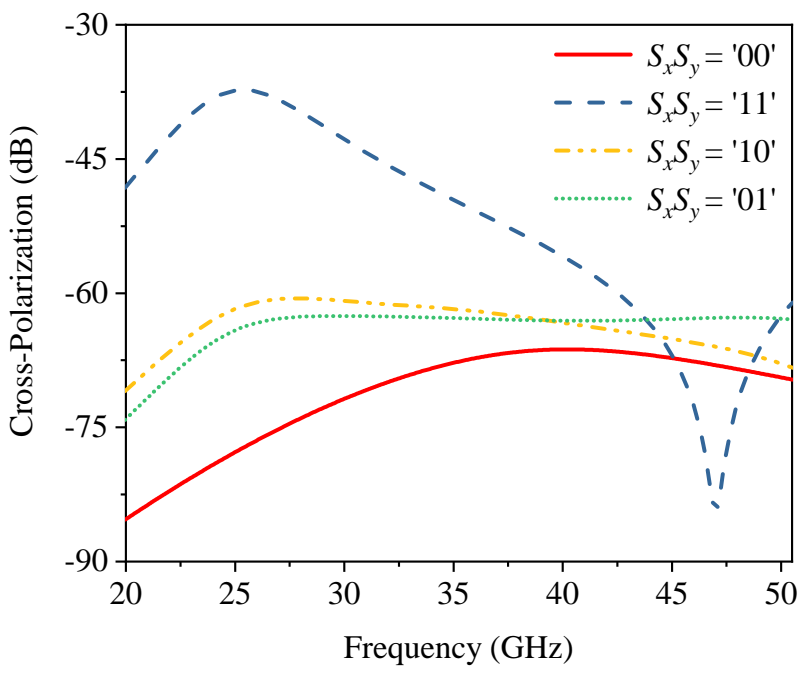

Fig. 5. Cross polarization for different states, under horizontally polarized waves.

$27.2 \mathrm{GHz}$ to $51.1 \mathrm{GHz}$, and $180 \pm 20$-degree reflective phase difference can be achieved over this bandwidth. The low crosspolarization levels for different states ensure the high isolation for the dual-polarized unit cell, as shown in Fig. 5 . Due to the symmetrical structure, a similar performance can be realized for vertically polarized waves, by controlling the states of $S_{y}$.

The proposed independently controllable dual-polarized 1bit unit cell with a simple single-layer structure, wide bandwidth and low cross-polarization performance indicates that independently controllable 1-bit dual-beam dual-polarized reflectarray antenna can be achieved, which will be discussed in Section III.

\section{REFLECTARRAY ANTENNA DESIGN}

Using the proposed unit cells, the independently controllable 1-bit dual-beam dual-polarized reflectarray antenna is then designed. A dual-linearly-polarized horn antenna with two ports is used as the feeding antenna, which can represent two 


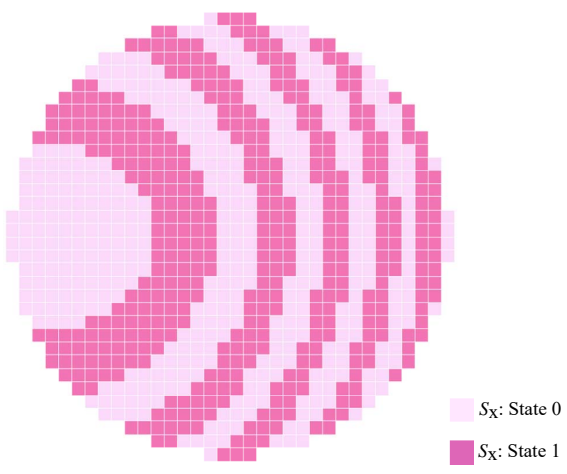

(a)

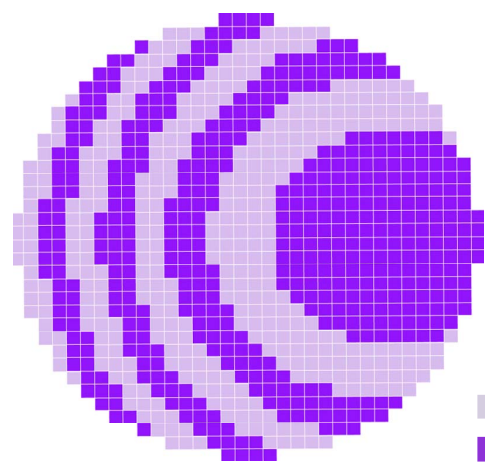

(b)

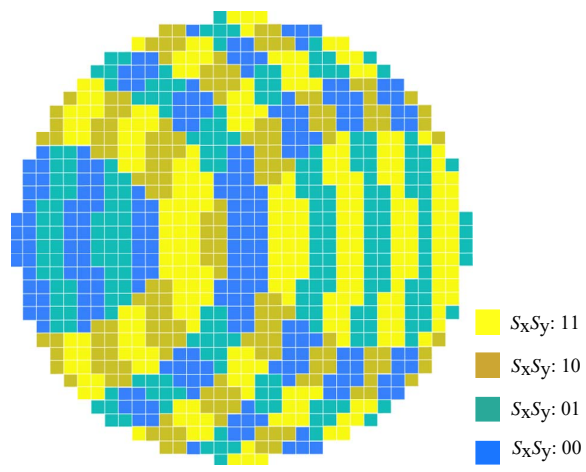

(c)

Fig. 6. State distributions: (a) State distributions for horizontal polarization; (b) State distributions for vertical polarization; (c) State distributions on the reflective metasurface.

ports of MU-MIMO systems. The gain of the horn antenna is about $14.5 \mathrm{dBi}$ with a $3-\mathrm{dB}$ beamwidth of 25.1 degrees at 33.0 GHz. The diameter of the array is $131.2 \mathrm{~mm}$, about $14.4 \lambda_{0}$, where $\lambda_{0}$ is the free-space wavelength at the frequency of 33 GHz. The total number of the unit cells is 864 , and the intercell spacing is $0.43 \lambda_{0}$. The horn antenna is positioned above the center of the reflective metasurface, and the aperture of the horn is parallel to the metasurface. The ratio of focal length to aperture diameter (F/D) is set as 1.0 in the reflectarray antenna design.

When the positions of the horn antenna and the metasurface are determined, the initial phase on the reflective surface causing by spherical incident waves of the horn antenna can be extracted with the help of CST Microwave Studio. Let $\theta$ and $\varphi$ denote the elevation angle and the azimuth angle, respectively. The targeted beam directions for the horizontally polarized and vertically polarized beam are $\theta_{\mathrm{H}}=-15^{\circ}$, $\varphi_{\mathrm{H}}=0^{\circ}$ and $\theta_{\mathrm{V}}=20^{\circ}, \varphi_{\mathrm{V}}=0^{\circ}$, respectively. The precise phase compensation $\psi_{\mathrm{H}, m n}$ of the $m$-th column and $n$-th row ( $m, n=1,2, \ldots)$ unit cell for the horizontally polarized beam can be calculated by

$$
\psi_{\mathrm{H}, m n}=-\frac{2 \pi m d}{\lambda_{0}} \sin \theta_{\mathrm{H}}+\Delta \phi_{\mathrm{H}}+\varphi_{\mathrm{H}, m n}
$$

where $\varphi_{\mathrm{H}, m n}$ is the initial phase of the $\{m, n\}$-th unit cell for horizontal polarization, $d$ is the spacing between the unit cells, $\Delta \phi_{\mathrm{H}}$ is a reference phase to provide an additional degree of freedom for the reflectarray design, which can be chosen from 0 to $2 \pi$ and has a significant effect on the sidelobe level (SLL) [18], [19]. The 1-bit phase compensation of the $\{m, n\}$-th unit cell for horizontal polarization can be given by

$$
\Phi_{\mathrm{H}, m n}=\left\{\begin{aligned}
\frac{\pi}{2} & \bmod \left(\psi_{\mathrm{H}, m n}, 2 \pi\right) \in(0, \pi], \\
\frac{3 \pi}{2} & \bmod \left(\psi_{\mathrm{H}, m n}, 2 \pi\right) \in(\pi, 2 \pi]
\end{aligned}\right.
$$

where $\bmod \left(\psi_{\mathrm{H}, m n}, 2 \pi\right)$ means the modulo of the precise phase compensation dividing $2 \pi$. The phase of $\frac{\pi}{2}$ and $\frac{3 \pi}{2}$ can be mapped to State 0 and State 1 of $S_{x}$, respectively, as shown in Fig. 6(a). Using the same method, the state of $S_{y}$ for vertical polarization can also be determined, as shown in Fig. 6(b). Then the state distributions of all unit cells on the reflective metasurface can be obtained, as shown in Fig. 6(c). The beam directions for horizontal and vertical polarizations

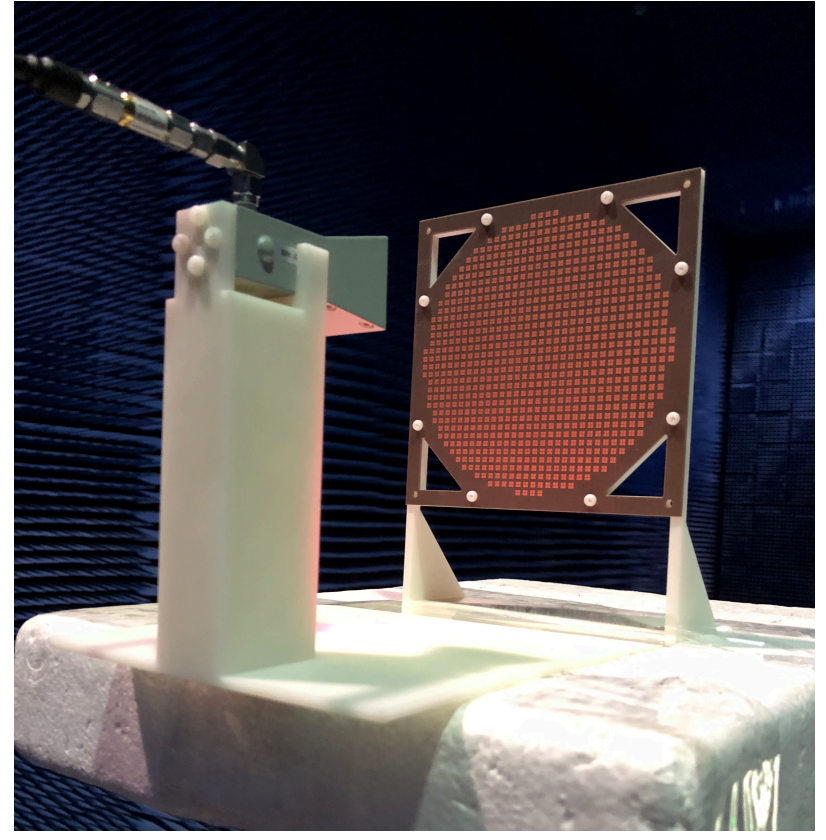

Fig. 7. Photograph of the fabricated independently controllable 1-bit dualbeam dual-polarized reflectarray antenna.

can be independently controlled by adjusting the dark grey stubs $\left(S_{x}\right)$ and the light grey stubs $\left(S_{y}\right)$ of the unit cells.

\section{RESUlts AND Discussions}

\section{A. Measurement Results}

The designed 1-bit dual-beam dual-polarized reflective metasurface is fabricated and measured. The photograph of the fabricated reflectarray antenna with its fixture is shown in Fig. 7. The feeding horn antenna and the reflective metasurface are assembled using some plastic screws and a 3D-print fixture. The radiation patterns and the gain of the reflectarray prototype are verified in the far-field anechoic chamber.

The simulated and measured far-field performances of the designed reflectarray with the feeding horn are compared in Figs. 8 and 9. Fig. 8 shows the normalized radiation patterns in the xoz-plane. The undistorted pencil-shaped beams are realized from $31.0 \mathrm{GHz}$ to $35.0 \mathrm{GHz}$ with the measured SLL 


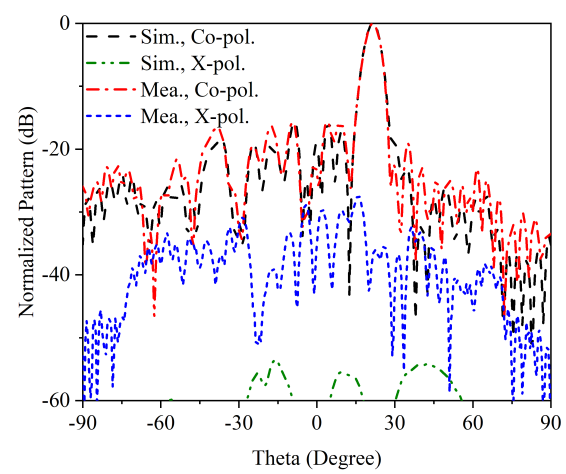

(a)

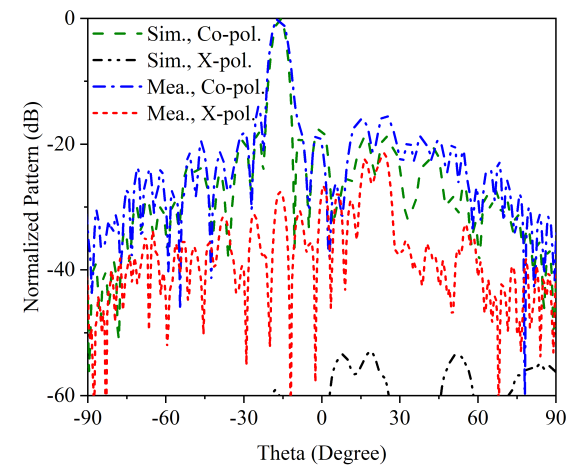

(d)

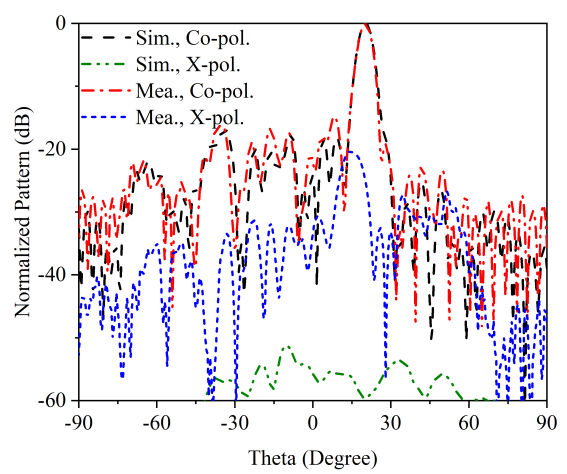

(b)

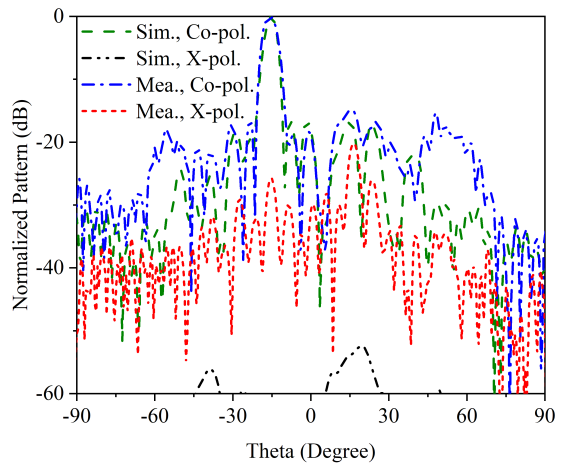

(e)

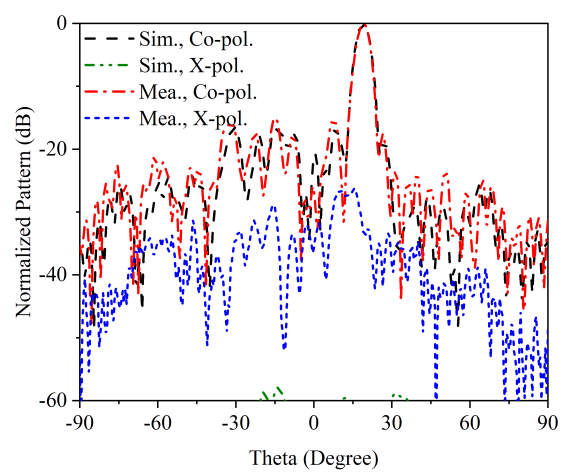

(c)

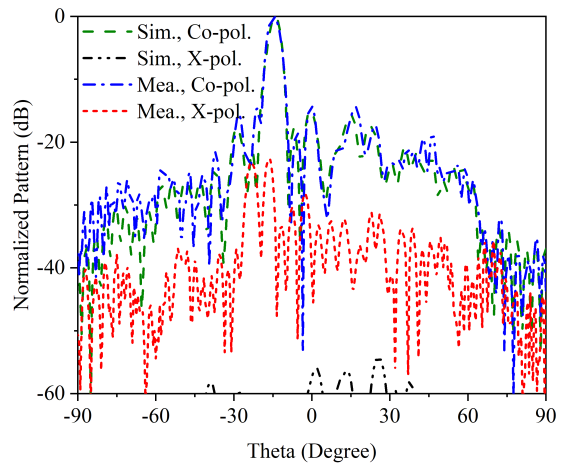

(f)

Fig. 8. Simulated and measured normalized radiation patterns with horizontally polarized incident waves: (a) $31.0 \mathrm{GHz}$; (b) $33.0 \mathrm{GHz}$; (c) $35.0 \mathrm{GHz}$. Vertically polarized incident waves: (d) $31.0 \mathrm{GHz}$; (e) $33.0 \mathrm{GHz}$; (f) $35.0 \mathrm{GHz}$.

less than $-14 \mathrm{~dB}$ for both polarizations. The measured SLLs are higher than the simulated ones with the influences of the fixture and the cables. The fluctuation of gains are less than $1 \mathrm{~dB}$ and the skewing of the beam directions are less than 1 degrees during this bandwidth. The measured crosspolarization of both beams are below $-20 \mathrm{~dB}$, indicating high polarization isolation.

As shown in Fig. 9, the measured 1.5-dB gain bandwidth and $2-\mathrm{dB}$ gain bandwidth are above $20.6 \%$ and $28.6 \%$, respectively, for both polarizations. The measured gain at $33.0 \mathrm{GHz}$ for the horizontal polarization and the vertical polarization are $25.0 \mathrm{dBi}$ and $25.8 \mathrm{dBi}$, respectively. The simulated and measured results agree well with the simulated results, demonstrate the independent polarization control performance and beamforming potential using the proposed 1-bit dual-polarized unit cell.

\section{B. Comparisons and Discussions}

Table III compares the proposed independently controllable 1-bit dual-beam dual-polarized reflectarray antenna with reported dual-linearly-polarized reflectarray antennas. For duallinearly-polarized unit cell design, the rectangle patches and dipoles are commonly used and the different reflective phases of dual polarization can be achieved by changing orthogonal geometrical dimensions. The multi-layer structure is utilized to realize sufficient reflective phase range, broaden the bandwidth of the unit cell, and some unit cells control different polarization on the different layers. However, for single-layer unit cells, it is difficult to realize independent dual-polarization

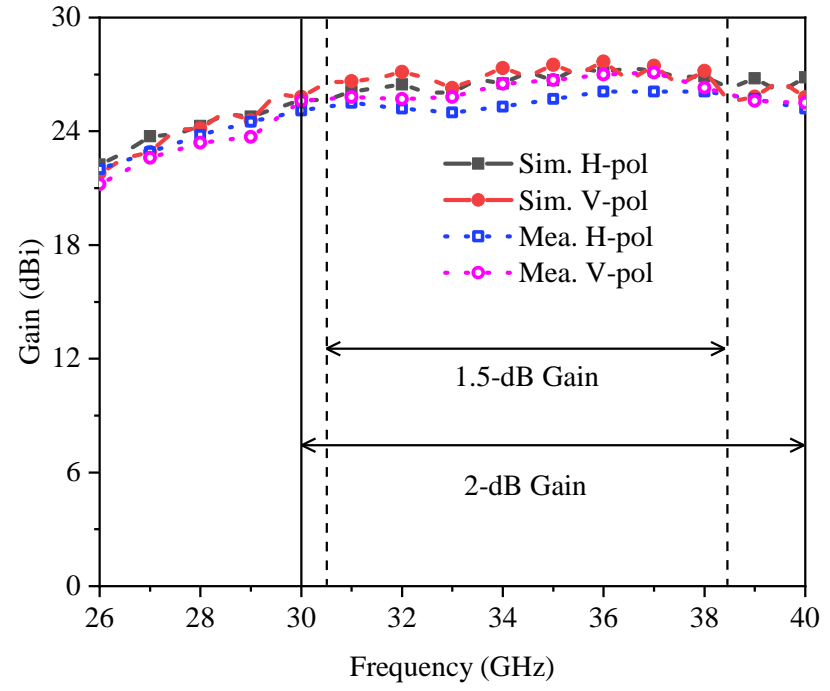

Fig. 9. Simulated and measured gains of the proposed independently controllable 1-bit dual-beam dual-polarized reflectarray antenna.

control with broadband performance. Thanks to the simple 1-bit phase compensation, the proposed unit cell has the advantages in wide bandwidth, single-layer structure and high polarization isolation. The proposed 1-bit unit cell achieves the bandwidth of $62.1 \%$, wider than reported unit cells.

The independently controllable 1-bit dual-beam dualpolarized reflectarray antenna can be achieved using the proposed unit cell. Compared with reported multi-layer independently controllable dual-polarized dual-beam reflectarray 
TABLE III

Comparisons of the Proposed Dual-Beam Dual-Polarized Reflectarray Antenna with Those in References.

\begin{tabular}{cccccccc}
\hline \hline Ref. & Unit cell & Pol. control & Layers num. & BW of unit cell & BW of RA & Compensation & Freq. (GHz) \\
\hline$[7]$ & Patch & Independent & 3 & N.G. & $10 \%(1-\mathrm{dB})$ & Precise & $11-12$ \\
\hline$[10]$ & Dipole & Independent & 2 & $\geq 24 \%$ & $27 \%(2-\mathrm{dB})$ & Precise & $11-14$ \\
\hline$[11]$ & Dipoles & Independent & 2 & $\geq 24 \%$ & $24 \%$ & Precise & $11-14$ \\
\hline$[20]$ & Loop+Patch+Dipole & Independent & 2 & N.G. & N.G. & 3 bit & $36 / 94$ \\
\hline$[21]$ & Patch & Dependent & 2 & N.G. & $16.7 \%(1.5-\mathrm{dB})$ & Precise & 11.95 \\
\hline$[22]$ & Cross loop & Dependent & 1 & $25 \%$ & $18 \%(1.5-\mathrm{dB})$ & Precise & 32 \\
\hline \multirow{2}{*}{ This work } & \multirow{2}{*}{ Cross-slot patch } & Independent & 1 & $62.1 \%$ & $\begin{array}{c}20.6 \%(1.5-\mathrm{dB}) \\
28.6 \%(2-\mathrm{dB})\end{array}$ & \multirow{2}{*}{1 bit } & 33 \\
\hline \hline
\end{tabular}

* N.G. represents "Not Given”.

antennas and single/multi-layer dependently controllable dualpolarized dual-beam reflectarray antennas, the proposed 1bit reflectarray antenna has the advantage in independently controllable dual-polarized dual beams, a single-layer structure and has a comparable gain bandwidth. Furthermore, the compact 1-bit phase compensation method, the single-layer structure, and the broadband unit cell bring a potential for proposed independently controllable 1-bit dual-beam dualpolarized reflectarray antenna to achieve dual-beam scanning capability.

\section{CONCLUSIONS}

An independently controllable 1-bit dual-polarized unit cell with broad phase bandwidth, high polarization isolation, and simple single-layer structure has been proposed. By manipulating the magnetic resonance frequency and the electrical resonance frequency of the two states, the 1-bit reflective phase of horizontally and vertically polarized EM waves have been independently controlled. Using the proposed unit cells, the independently controllable 1-bit dual-polarized dualbeam reflectarray antenna have been designed, simulated, and fabricated. The proposed dual-beam reflectarray antenna can independently control horizontally and vertically polarized beams to desired directions. The achieved performance of the proposed reflectarray antenna has demonstrated the potential for MU-MIMO applications.

\section{REFERENCES}

[1] T. L. Marzetta, Fundamentals of massive MIMO. Cambridge University Press, 2016.

[2] O. Jo, J.-J. Kim, J. Yoon, D. Choi, and W. Hong, "Exploitation of dual-polarization diversity for $5 \mathrm{G}$ millimeter-wave MIMO beamforming systems," IEEE Trans. Antennas Propag., vol. 65, no. 12, pp. 66466655, 2017.

[3] G. Moreno, A. B. Yakovlev, H. M. Bernety, D. H. Werner, H. Xin, A. Monti, F. Bilotti, and A. Alu, "Wideband elliptical metasurface cloaks in printed antenna technology," IEEE Trans. Antennas Propag., vol. 66, no. 7 , pp. $3512-3525,2018$.

[4] K. Chen, G. Ding, G. Hu, Z. Jin, J. Zhao, Y. Feng, T. Jiang, A. Alù, and C.-W. Qiu, "Directional janus metasurface," Advanced Materials, $\mathrm{p}$. 1906352, 2019.

[5] O. Yurduseven and D. R. Smith, "Dual-polarization printed holographic multibeam metasurface antenna," IEEE Trans. Antennas Propag., vol. 16, pp. 2738-2741, 2017.

[6] X. Zhang, Q. Yu, W. Jiang, Y. Sun, L. Bai, Q. Wang, C.-W. Qiu, and T. Cui, "Polarization-controlled dual-programmable metasurfaces," Advanced Science, p. 1903382, 2020.
[7] J. A. Encinar, L. S. Datashvili, J. A. Zornoza, M. Arrebola, M. SierraCastañer, J. L. Besada-Sanmartin, H. Baier, and H. Legay, "Dualpolarization dual-coverage reflectarray for space applications," IEEE Trans. Antennas Propag., vol. 54, no. 10, pp. 2827-2837, 2006.

[8] R. Javor, X.-D. Wu, and K. Chang, "Dual polarisation of microstrip reflectarray antenna," Electro. lett., vol. 30, no. 13, pp. 1018-1019, 1994.

[9] H. Hasani, C. Peixeiro, A. K. Skrivervik, and J. Perruisseau-Carrier, "Single-layer quad-band printed reflectarray antenna with dual linear polarization," IEEE Trans. Antennas Propag., vol. 63, no. 12, pp. 5522 $5528,2015$.

[10] R. Florencio, J. A. Encinar, R. R. Boix, V. Losada, and G. Toso, "Reflectarray antennas for dual polarization and broadband telecom satellite applications," IEEE Trans. Antennas Propag., vol. 63, no. 4, pp. 1234-1246, 2015.

[11] J. A. Encinar, R. Florencio, M. Arrebola, M. A. S. Natera, M. Barba, J. E. Page, R. R. Boix, and G. Toso, "Dual-polarization reflectarray in Ku-band based on two layers of dipole arrays for a transmit-receive satellite antenna with south american coverage," International Journal of Microw. and Wireless Technol., vol. 10, no. 2, pp. 149-159, 2018.

[12] Z. H. Jiang, T. Yue, and W. Hong, "Low-profile and wideband dualcircularly-polarized reflect-arrays based on rotated mtal-backed dualpolarized aperture-coupled patch elements," IEEE Trans. Antennas Propag., vol. 68, no. 3, pp. 2108-2177, 2019.

[13] W.-L. Guo, G.-M. Wang, W.-Y. Ji, Y.-L. Zheng, K. Chen, and Y. Feng, "Broadband spin-decoupled metasurface for dual-circularly polarized reflector antenna design," IEEE Trans. Antennas Propag., vol. 68, no. 5, pp. 3534-3543, 2020

[14] W. Pan, C. Huang, X. Ma, B. Jiang, and X. Luo, "A dual linearly polarized transmitarray element with 1-bit phase resolution in X-band," IEEE Antennas Wireless Propag. Lett., vol. 14, pp. 167-170, 2014.

[15] T. Debogovic and J. Perruisseau-Carrier, "Low loss MEMSreconfigurable 1-bit reflectarray cell with dual-linear polarization," IEEE Trans. Antennas Propag., vol. 62, no. 10, pp. 5055-5060, 2014.

[16] H. Yang, F. Yang, S. Xu, M. Li, X. Cao, and J. Gao, "A 1-bit multipolarization reflectarray element for reconfigurable large-aperture antennas," IEEE Antennas Wireless Propag. Lett., vol. 16, pp. 581-584, 2016.

[17] J. Yin, Q. Wu, Q. Lou, H. Wang, Z. N. Chen, and W. Hong, "Singlebeam 1-bit reflective metasurface using pre-phased unit cells for normally incident plane waves," IEEE Trans. Antennas Propag., pp. 1-1, 2020.

[18] Y. Mao, S. Xu, F. Yang, and A. Z. Elsherbeni, "A novel phase synthesis approach for wideband reflectarray design," IEEE Trans. Antennas Propag., vol. 63, no. 9, pp. 4189-4193, 2015.

[19] H. Yang, F. Yang, S. Xu, Y. Mao, M. Li, X. Cao, and J. Gao, "A 1-bit $10 \times 10$ reconfigurable reflectarray antenna: design, optimization, and experiment," IEEE Trans. Antennas Propag., vol. 64, no. 6, pp. 22462254, 2016.

[20] Q. Wang, Z. H. Shao, Y. J. Cheng, and P. K. Li, "Ka/W dualband reflectarray antenna for dual linear polarization," IEEE Antennas Wireless Propag. Lett., vol. 16, pp. 1301-1304, 2016.

[21] J. A. Encinar, "Design of two-layer printed reflectarrays using patches of variable size," IEEE Trans. Antennas Propag., vol. 49, no. 10, pp. 1403-1410, 2001.

[22] Q. Wang, Z. Shao, P. Li, L. Li, and Y. Cheng, "A dual polarization, broadband, millimeter-wave reflectarray using modified cross loop element," Microw. Opt. Technol. Lett., vol. 56, no. 2, pp. 287-293, 2014. 